\title{
Monte Carlo Simulations of Proton Acceleration in Colliding Wind Binaries
}

\section{E. Grimaldo*}

Institute for Theoretical Physics, University of Innsbruck, Austria

E-mail: emanuele.grimaldo@uibk.ac.at

\section{A. Reimer}

Institute for Theoretical Physics, University of Innsbruck, Austria

E-mail: anita.reimerauibk.ac.at

\section{R. Kissmann}

Institute for Astro- and Particle Physics, University of Innsbruck, Austria

E-mail: ralf.kissmann@uibk.ac.at

\begin{abstract}
Particles accelerated at the shocks forming at the wind collision region of a binary system of massive stars are expected to produce $\gamma$-rays predominantly either through inverse Compton scattering of electrons in the stellar radiation fields, or through the decay of neutral pions produced in proton-proton collisions. Up to now, the only colliding wind binary (CWB) system associated with $\gamma$-ray emission is $\eta$ Carinae, where two components seem to be present. So far, there is no evidence for $\gamma$-ray emission from other binary massive star systems, such as WR 140 or WR 147 , which were expected to be detected with comparable or even higher fluxes. We investigate injection and acceleration of protons in a typical CWB system by means of Monte Carlo simulations with a test-particle approach. We rely on hydrodynamic simulations for determining the background conditions in the wind collision region. Both shocks on either side of the contact discontinuity are considered, looking for different accelerated particle populations, that could result in different components of the $\gamma$-ray spectrum. Such studies may contribute to understand the lack of detection of $\gamma$-rays from most of these CWB systems up to now.
\end{abstract}

The 34th International Cosmic Ray Conference,

30 July- 6 August, 2015

The Hague, The Netherlands

\footnotetext{
* Speaker.
} 


\section{Introduction}

It is well known that particle acceleration occurs in environments where a collisionless plasma forms a shock front, resulting in a power law energy spectrum of the non-thermal population (e.g. $[1,2]$ and references therein). Due to their features, Colliding Wind Binaries (CWBs) are expected to be systems where particle acceleration takes place. In fact, a CWB consists of a binary system of massive, hot stars with strong stellar winds. Shocks form at the Wind Collision Region (WCR), thus creating a suitable environment for accelerating particles by means of the so-called Diffusive Shock Acceleration (DSA). Besides the detected radio synchrotron radiation $[3,4,5]$ from such systems, processes such as inverse Compton (IC) scattering, relativistic Bremsstrahlung, and $\pi^{0}$ decay are expected to provide sufficiently high fluxes of $\mathrm{GeV}$ and $\mathrm{TeV} \gamma$-rays to allow detection by instruments such as Fermi-LAT, HESS, MAGIC and VERITAS (e.g. [6], [7], [8]). In contrast to such predictions, $\gamma$-ray emission from CWBs has not been observed up to now, with the exception of $\eta$ Carinae [9, 10, 11].

Aiming at achieving a better understanding of these systems, hydrodynamic (HD) models of CWBs have been developed, which combine three-dimensional HD numerical simulations with the solution of the transport equation of high-energy particles, yielding their spectral energy distributions for typical CWB systems [12]. Based on these distributions, the high-energy non-thermal photon emission has been studied [13]. However, in these studies, particle acceleration is modelled in the transport equation by adding a very simplified term to the energy gain, which is the analytical result obtained when considering a population of suprathermal particles at a shock of infinite extent. This approach does not allow to study the injection efficiency of thermal particles into the acceleration process, i.e. how many of the particles from the thermal distribution are able to recross the shock from downstream to upstream.

Different methods have been used to simulate particle acceleration and to investigate injection efficiency at collisionless shocks, such as (full) particle in cell (PIC), hybrid PIC, and Monte Carlo simulations. In full and hybrid PIC simulations, particles move in the simulation box and are scattered by self-consistently generated magnetic turbulences (e.g. [14, 15] and references therein). In the Monte Carlo approach, on the other hand, magnetic fields are predefined: particles move in the simulation region, and interact with the background when a scattering occurs. The scattering process is necessarily modelled.

Although ideal for studying the microphysics of shock formation and particle injection into the acceleration process from first principles, PIC simulations are computationally very demanding, and both box sizes and time intervals that can be investigated are limited. Monte Carlo simulations, relying on modelled scattering, can extend over much larger regions and time ranges (and therefore energies). Since we want to consider a large extent of the WCR, and are mainly interested in energies much larger than the thermal energy, we chose the Monte Carlo method for our purpose. In particular, we employ a test-particle approach, closely resembling methods used for studying particle injection at oblique shocks (e.g. $[16,17,18]$ ). In order to obtain more realistic background conditions, we rely on hydrodynamic (HD) simulations of a typical CWB system. In section 2, we describe the method employed for this work. Details of the system studied, as well as the results of the simulations, can be found in section 3. Finally, section 4 is devoted to the conclusions. 


\section{Description of the method}

In the most basic set up, the simulation volume is divided only into two cells, corresponding to the upstream and the downstream regions. Each cell is characterized by its background conditions: plasma flow velocity, magnetic field, temperature, and density. These are initialized at the beginning of the simulation and, since we are applying a test-particle approach, they are not changed by the accelerated particles. Protons are injected into the system with a Maxwell-Boltzmann distribution of velocities, at the temperature of the underlying plasma, and are followed in a frame where the shock front is stationary. The pitch angle distribution is isotropic in the frame comoving with the local plasma flow. The particles, the positions of which, in the present work, are approximated by their guiding centers, move undisturbed, until: (i) a scattering occurs, (ii) the particle reaches the boundary of a cell, or (iii) the particle reaches the edge of the simulation region. We assume the scattering to be elastic in the local plasma frame, and to isotropize the pitch angle of particles in a single event, which models large fluctuations in the magnetic field [20].

Cross-field diffusion is modelled by randomly moving the guiding center a distance between 0 and $2 r_{g}$ perpendicular to the magnetic field lines, with $r_{g}=p \sin \theta /(q B)$, where $p$ is the momentum of the particle, $\theta$ is the pitch angle, $q$ is the charge of the particle, and $B$ is the magnetic field in the appropriate cell [21].

The time between scatterings is exponentially distributed, with mean value $t_{c}=\eta \tilde{r}_{g} / v$, where $\eta$ is a proportionality factor, $v$ is the speed of the particle, and $\tilde{r}_{g}=p / q B$ [20].

When a particle reaches the boundary of a cell, we assume that it conserves its magnetic moment. Therefore, a particle is either reflected at the boundary, or transmitted with a new, appropriate pitch angle.

Particles are removed from the system when they reach the boundary of the simulation region.

In order to improve statistics at higher energies, the technique of particle splitting is employed: a statistical weight is assigned to each particle at the beginning of the simulation. When a particle is removed from the system, one of the remaining particles is splitted, i.e. a particle with (temporarily) identical position and momentum is created, while the statistical weight is halved. The spectrum of the flux through a certain surface is recorded by adding to the appropriate energy bin the statistical weight of the particle crossing it. With our code, under this basic setup, we can reproduce the results obtained in Ref. [16].

As already mentioned, we want to investigate particle acceleration in the wind collision region of CWBs. For this purpose, the background cells (in this case more than two) are initialized with the results of HD simulations of a typical CWB system, carried out using the CRONOS code [22, 23]. In the HD simulations, the transition region of the shock front is about three cells wide for numerical reasons only. Therefore, we cut these three cells when initializing the background for the Monte Carlo simulations.

In the present work, the magnetic field lines are assumed to be aligned to the velocity of the flowing plasma. 


\section{Results}

\subsection{Parameters of the system}

The system under study is the same as in Reitberger et al. (2014) [12]. It consists of a B star and a Wolf-Rayet star, with the parameters listed in Table 1 . The stellar separation is $R=720 \mathrm{R} \odot$.

\begin{tabular}{lccccccccc}
\hline Star & $\begin{array}{c}M_{*} \\
{\left[\mathrm{M}_{\odot}\right]}\end{array}$ & $\begin{array}{c}R_{*} \\
{\left[\mathrm{R}_{\odot}\right]}\end{array}$ & $\begin{array}{c}T_{*} \\
{[\mathrm{~K}]}\end{array}$ & $\begin{array}{c}L_{*} \\
{\left[\mathrm{~L}_{\odot}\right]}\end{array}$ & $\begin{array}{c}\dot{M} \\
{\left[\mathrm{M}_{\odot} \mathrm{yr}^{-1}\right]}\end{array}$ & $\begin{array}{c}v_{\infty} \\
{\left[\mathrm{km} \mathrm{s}^{-1}\right]}\end{array}$ & $\alpha$ & $k$ & $\beta$ \\
\hline B & 30 & 20 & 23000 & $10^{5}$ & $10^{-6}$ & 4000 & 0.642 & 0.5 & 0.83 \\
WR & 30 & 10 & 40000 & $2.3 \times 10^{5}$ & $10^{-5}$ & 4000 & 0.678 & 0.7 & 0.83 \\
\hline
\end{tabular}

Table 1: Stellar and wind parameters of a typical colliding wind binary system, taken from [12]. $M_{*}$ is the stellar mass, $R_{*}$ the stellar radius, $T_{*}$ the effective temperature, $L_{*}$ the luminosity, $\dot{M}$ the mass loss rate, $v_{\infty}$ the terminal velocity of the wind, and $\alpha, k$ and $\beta$ are the CAK parameters $[25,26]$.

An example of the results of a HD simulation can be seen in Fig. 1. The blue square highlights the selected region used in the Monte Carlo simulations, which consists of $(60 \times 60 \times 60)$ cells. For the

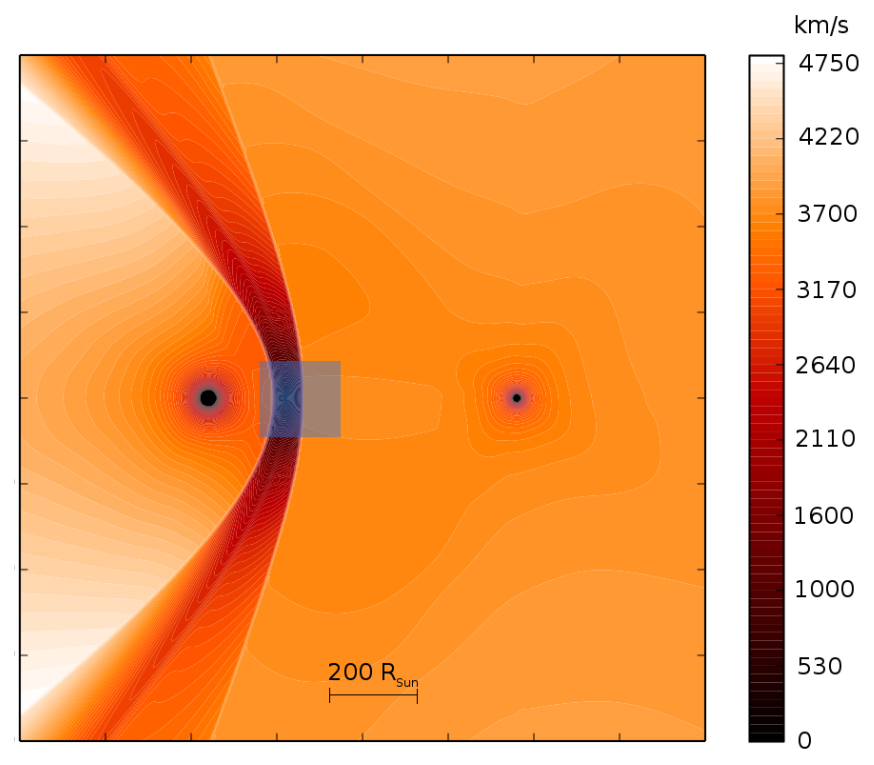

Figure 1: Absolute plasma flow velocity map of a colliding wind binary system (hydrodynamic simulation). The wind collision region is located between the B star (left) and the WR star (right), and is delimited by two shocks. The blue square highlights the region selected for the Monte Carlo simulation.

cells directly upstream of the two shock fronts, the strength (but not the topology) of the magnetic field is determined following the approximation employed by Usov and Melrose (1992) [27]. At the shocks, we apply the magnetohydrodynamic shock-jump conditions to obtain the field in the cells directly downstream. Starting from these cells, inside the WCR, we assume the magnetic field to be proportional to the thermal energy density of the underlying plasma [29]. The fields on 
either side of the WCR are thus scaled from the shock fronts up to the contact discontinuity. Here, the magnetic field is also discontinuous, assuming the surface magnetic field of the two stars to be $B_{*_{W R}}=B_{*_{B}} \approx 100 \mathrm{G}$. MHD simulations of colliding wind binaries point towards the existence of such a field discontinuity [28].

Particles are injected in cells directly upstream of the shock fronts on either side of the WCR, close to its apex. The fluxes are recorded at a surface tangent to the shock front, close to the injection cells. Here, we show the results of simulations carried out with $\eta=3$ (highly turbulent medium) [20]. Investigating the results for different values of $\eta$ is subject of future studies.

\subsection{Spectra}

An analytical treatment of the acceleration process yields a flux of accelerated particles $J \propto\left(E\left(E+2 m_{p} c^{2}\right)\right)^{-\frac{\sigma}{2}}$, where $E$ is the kinetic energy, $m_{p}$ is the mass of the proton, and $\sigma=(r+2) /(r-1)$ is the spectral index, with the compression ratio $r$. The spectra resulting from the Monte Carlo simulations can be seen in Figure 2. The dashed and dotted lines represent the

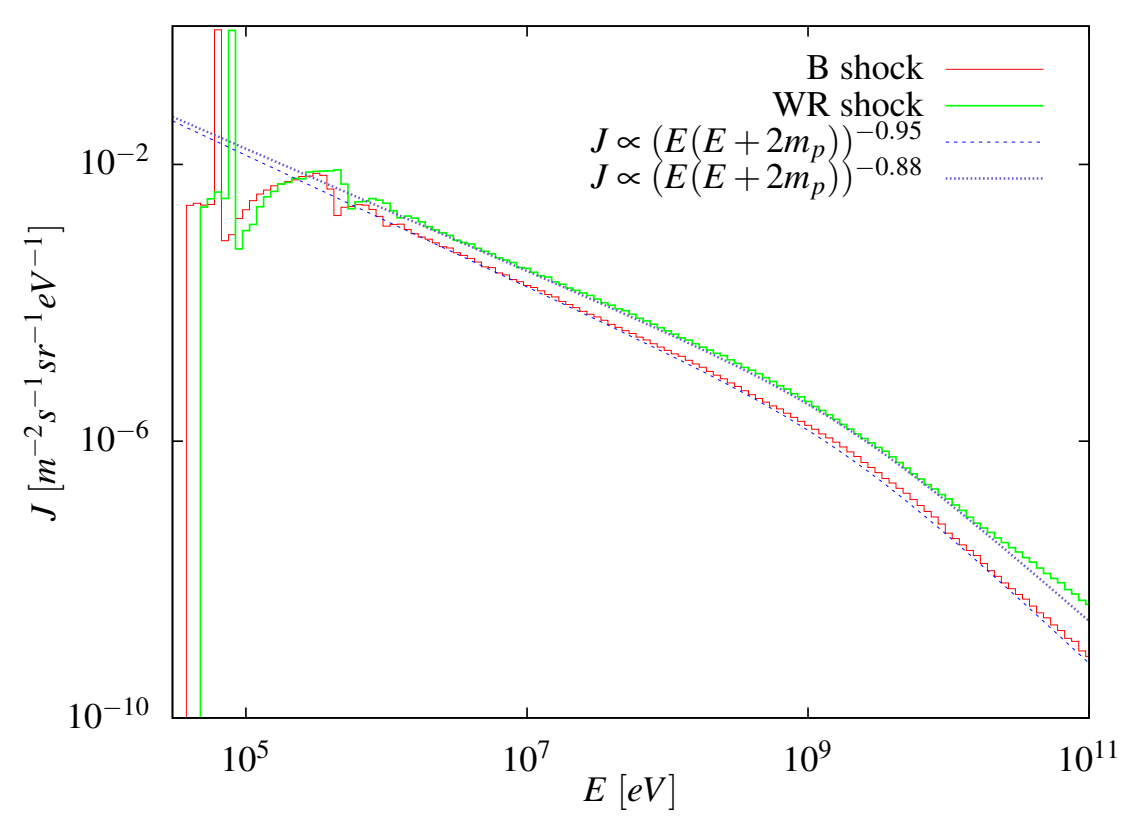

Figure 2: Fluxes of protons towards the inner part of the wind collision region. The particles are injected at the shocks on the B-side and on the WR-side of the WCR. The spectra are recorded at the corresponding shock front.

best fits to the non-thermal tails, corresponding to $\sigma_{B}=1.90 \pm 0.02$ for the B-side shock, and $\sigma_{W R}=1.76 \pm 0.02$ for the WR-side shock. The difference between the two spectral indices can be ascribed to slightly different compression ratios on the two sides of the WCR. In fact, in the region we are considering, we have $r_{W R}>r_{B}>4$, where $r_{W R}$ and $r_{B}$ are the compression ratios of the shock on the WR side and on the B side, respectively.

With the parameters used in this work, up to $100 \mathrm{GeV}$, we do not find any effects of the doubleshock structure of the WCR. Nevertheless, these might be observable at higher energies or, within the energy range considered here, by reducing the strength of the magnetic fields or by increasing 
$\eta$. In fact, these two changes would result in larger mean free paths of the particles. This, in turn, might increase their probability of reaching the other shock front, once crossed the contact discontinuity, before being advected beyond the boundary of the simulated region. This will be subject of future studies.

The Monte Carlo method also allows to estimate the efficiency of injection of particles into the acceleration process, dependent on the geometrical set up of the system. Although relying to some extent on the assumptions made when modelling the scattering process, this estimate, which cannot be done in analytical treatments of particle acceleration, may be useful for improving the models aiming at studying the non-thermal $\gamma$-ray emission of CWB systems. If we define the injection efficiency $\varepsilon$ as the ratio between the fluxes of thermal to non-thermal particles, we obtain $\varepsilon_{W R} \approx 14 \%$ for the WR shock, and $\varepsilon_{B} \approx 11 \%$ for the B shock. This small difference can also be understood as an effect of the discrepancy between the compression ratios at the two shock fronts: with higher compression ratios, thermal particles crossing the shock from upstream have higher velocities with respect to the scattering centers downstream, and they are advected away less efficiently. Here, we consider a region where the shocks are quasiparallel. Regions farther away from the apex of the WCR have increasing obliquity, and the injection efficiency is expected to decrease $[16,18,15]$.

\section{Conclusions}

In the presented work, we investigated, by means of Monte Carlo simulations, the acceleration of protons in a typical colliding wind binary system. Our code is able to reproduce previous results of particle acceleration at oblique shocks, in its most basic setup (two-cells acceleration region with flat shock of effective infinite extent). One of the most interesting features of our approach is the use of hydrodynamical simulations of CWB systems, which have been exploited for determining the background of the test particle Monte Carlo simulations, allowing us to consider more realistic environments. Under the assumptions introduced in Sections 2 and 3 for the scattering of protons and for the magnetic field, we found a slight difference between the spectral index of the fluxes of particles accelerated at the shock front on the B side of the WCR, and the one referred to the shock on the WR side. This can be explained, considering that the compression ratio on the $\mathrm{B}$ side is slightly lower than that on the WR side. Nevertheless, such a discrepancy does not necessarily correspond to two observable components in the gamma-ray spectrum: accelerated protons can produce $\gamma$-rays via nucleon-nucleon interactions, with the production and successive decay of neutral pions. A higher number of both target nuclei and accelerated protons in the system corresponds to a higher probability of interaction, therefore the densities of the two winds must be taken into account for determining the dominant component of accelerated particles. In this work, the two shocks delimiting the wind collision region are very similar. This is not always the case: a well known example is $\eta$ Carinae, where the winds have much different velocities and densities, especially close to periastron. $\eta$ Carinae is a very complex system, and a treatment such as the one described in this work is challenging. Also in other CWBs, the shock fronts delimiting the wind collision region may be considerably different, and it will be interesting to investigate such systems. Future work will also include exploring several combinations of parameters of scattering and magnetic fields. 


\section{Acknowledgements}

Emanuele Grimaldo and Anita Reimer acknowledge financial support from the Austrian Science Fund (FWF), project P 24926-N27. We also thank Klaus Reitberger for helpful discussions.

\section{References}

[1] F. C. Jones and D. C. Ellison, The plasma physics of shock acceleration, Space Science Reviews 58 (1991) 259-346

[2] L. O'C. Drury, An introduction to the theory of diffusive shock acceleration of energetic particles in tenuous plasmas, Reports on Progress in Physics 46 (1983) 973

[3] P. M. Williams et al., Radio and infrared structure of the colliding-wind Wolf-Rayet system WR 147, Monthly Notices of the Royal Astronomical Society 289 (1997) 10-20

[4] J. M. Chapman et al., Radio continuum measurements of southern early-type stars. III. Nonthermal emission from Wolf-Rayet Stars, The Astrophysical Journal 518 (1999) 890

[5] S. M. Dougherty et al., High-resolution radio observations of the colliding-wind binary WR 140, The Astrophysical Journal 623 (2005) 447

[6] D. Eichler and V. V. Usov, Particle acceleration and nonthermal radio emission in binaries of early-type stars, The Astrophysical Journal 402 (1993) 271-279

[7] A. Reimer, M. Pohl and O. Reimer, Nonthermal high-energy emission from colliding winds of massive stars, The Astrophysical Journal 644 (2006) 1118

[8] P. Benaglia and G. E. Romero, Gamma-ray emission from Wolf-Rayet binaries, Astronomy \& Astrophysics 399 (2003) 1121-1134

[9] M. Werner, O. Reimer, A. Reimer and K. Egberts, Fermi-LAT upper limits on gamma-ray emission from colliding wind binaries, Astronomy \& Astrophysics 555 (2013) A102

[10] K. Reitberger et al., Gamma-ray follow-up studies on $\eta$ Carinae, Astronomy \& Astrophysics 544 (2012) A98

[11] K. Reitberger et al., The first full orbit of $\eta$ Carinae seen by Fermi, Astronomy \& Astrophysics 577 (2015) A100

[12] K. Reitberger et al., High-energy Particle Transport in Three-dimensional Hydrodynamic Models of Colliding-wind Binaries, The Astrophysical Journal 782 (2014) 96

[13] K. Reitberger et al., Simulating three-dimensional nonthermal high-energy photon emission in colliding-wind binaries, The Astrophysical Journal 789 (2014) 87

[14] L. Gargaté and A. Spitkovsky, Ion Acceleration in Non-relativistic Astrophysical Shocks, The Astrophysical Journal 744 (2012) 67

[15] D. Caprioli and A. Spitkovsky, Simulations of Ion Acceleration at Non-relativistic Shocks. I. Acceleration Efficiency, The Astrophysical Journal 783 (2014) 91

[16] M. G. Baring, D. C. Ellison and F. C. Jones, The injection and acceleration of particles in oblique shocks - A unified Monte Carlo description, The Astrophysical Journal 409 (1993) 327-332

[17] M. G. Baring, D. C. Ellison and F. C. Jones, Monte Carlo simulations of particle acceleration at oblique shocks, Astrophysical Journal Supplement Series 90 (1994) 547-552 
[18] , M. G. Baring, D. C. Ellison and F. C. Jones, Monte Carlo simulations of particle acceleration at oblique shocks: Including cross-field diffusion, Advances in Space Research 15 (1995) 397-400

[19] D. C. Ellison, S. P. Reynolds and F. C. Jones, First-order Fermi particle acceleration by relativistic shocks, The Astrophysical Journal 360 (1990) 702-714

[20] D. C. Ellison, M. G. Baring and F. C. Jones, Acceleration Rates and Injection Efficiencies in Oblique Shocks, The Astrophysical Journal 453 (1995) 873

[21] F. C. Jones, M. G. Baring and D. C. Ellison, Modeling Cross Field Diffusion in Monte Carlo Calculations of Shock Acceleration, International Cosmic Ray Conference 2 (1993) 243

[22] R. Kissmann, J. Kleimann, H. Fichtner and R. Grauer, Local turbulence simulations for the multiphase ISM, Monthly Notices of the Royal Astronomical Society 391 (2008) 1577-1588

[23] J. Kleimann, A. Kopp, H. Fichtner and R. Grauer, A novel code for numerical 3-D MHD studies of CME expansion, Annales Geophysicae 27 (2009) 989-1004

[24] J. I. Castor, D. C. Abbott and R. I. Klein, Radiation-driven winds in Of stars, The Astrophysical Journal 195 (1975) 157-174

[25] J. I. Castor, D. C. Abbott and R. I. Klein, Radiation-driven winds in Of stars, The Astrophysical Journal 195 (1975) 157-174

[26] A. Pauldrach, J. Puls and R. P. Kudritzki, Radiation-driven winds of hot luminous stars Improvements of the theory and first results, Astronomy \& Astrophysics 164 (1986) 86-100

[27] V. V. Usov and D. B. Melrose, X-ray emission from single magnetic early-type stars, The Astrophysical Journal 395 (1992) 575-581

[28] A. Orgozałek, MHD simulations of colliding stellar winds in binary systems, Master Thesis, University of Innsbruck

[29] J. M. Pittard and S. M. Dougherty, Radio, X-ray, and $\gamma$-ray emission models of the colliding-wind binary WR140, Monthly Notices of the Royal Astronomical Society 372 (2006) 801-826 